\title{
Review of the Chinese leafhopper genus Parazyginella Chou \& Zhang (Hemiptera, Cicadellidae, Typhlocybinae, Zyginellini) with description of a new species
}

\author{
Xia Gao ${ }^{1,2, \dagger}$, Min Huang ${ }^{1, \ddagger}$, Yalin Zhang ${ }^{1, \S}$
}

I Key Laboratory of Plant Protection Resources and Pest Management, Ministry of Education, Entomological Museum (P.O. Box 55\#), College of Plant Protection, Northwest A \& F University, Yangling, Shaanxi 712100, Chinas 2 Hanzhong Agricultural Technology Extension Center, Hanzhong, Shaanxi 723000, China

† urn:lsid:zoobank.org:author:AF85701D-CE90-4A5F-B689-6A8661C1BABD

† urn:lsid:zoobank.org:author:59A337C4-OFE9-496F-880C-2BA94D4F203B

§ urn:lsid:zoobank.org:author:B4F3AAC7-28C2-4F9E-B055-7E772EB7A522

Corresponding author: Yalin Zhang (yalinzh@yahoo.com.cn)

Academic editor: Mick Webb | Received 9 October 2011 | Accepted 6 April 2012 | Published 19 April 2012

urn:lsid:zoobank.org:pub:EB495E55-668E-4128-9B08-654C0D643458

Citation: Gao X, Huang M, Zhang Y (2012) Review of the Chinese leafhopper genus Parazyginella Chou \& Zhang (Hemiptera, Cicadellidae, Typhlocybinae, Zyginellini) with description of a new species. ZooKeys 183: 17-22. doi: $10.3897 /$ zookeys. 183.2229

\begin{abstract}
The two leafhopper species in the genus Parazyginella Chou \& Zhang from China are reviewed and illustrated including one new species, Parazyginella tiani sp. n. which is described. A key to separate males of the two species is provided.
\end{abstract}

\section{Keywords}

Homoptera, Auchenorrhyncha, taxonomy

\section{Introduction}

Zyginellini is one of the smaller tribes in the large leafhopper subfamily Typhlocybinae. Members of the subfamily feed on trees, shrubs and herbs and some occur on economic crops including two species of Zyginellini, Zyginella mali (Yang) and Z. minuta (Yang), which damage apple trees in China. Members of this tribe can be distinguished by their usual bright coloration with distinct patterns and by the hindwing venation

Copyright Xia Gao et al. This is an open access article distributed under the terms of the Creative Commons Attribution License 3.0 (CC-BY), which permits unrestricted use, distribution, and reproduction in any medium, provided the original author and source are credited. 
with the vannal veins separate apically, with only one transverse vein visible and submarginal vein extended directly to vein $\mathrm{CuA}$, forming one open cell (Fig. 13).

The Oriental Zyginellini genus Parazyginella was erected by Chou and Zhang (1985) with $P$. lingtianensis from Guangxi, China as its type species. There have been no further reports of this genus which is recognized by its depressed body form and a dark spot near the apex of the forewing (Figs 1-3). In this paper, we describe a second species, Parazyginella tianisp. n. from Yunnan, China. The type specimens of the new species are deposited in the collections of the Entomological Museum, Northwest A \& F University, Yangling, China (NWAFU) and The Natural History Museum, London (BMNH).

\section{Systematics}

\section{Parazyginella Chou \& Zhang, 1985}

http://species-id.net/wiki/Parazyginella

Parazyginella Chou \& Zhang, 1985: 295; Zhang 1990: 174.

Type species. Parazyginella lingtianensis Chou \& Zhang, 1985

Description. Body flattened. Vertex conically produced, middle length nearly equal to width and length of pronotum; coronal suture distinct. Head and pronotum whitish yellow. Scutellum and venter yellow. Forewing with base of $1^{\text {st }}$ and $4^{\text {th }}$ apical cells at same level; $3^{\text {rd }}$ apical cell triangular and with a dark spot; hind margin of brochosome field and veins in apical area of wing touched with dark brown.

Abdominal apodemes elongate, reaching beyond 5th abdominal sternite.

Male pygofer strongly sclerotized, with short, finger-like process caudo-dorsally and few scattered microsetae. Subgenital plates broad at base, distally abruptly tapered to short narrow apex, with few macrosetae basally. Paramere simple, with central part expanded, apical part tapering to acute apex and strongly bent. Connective with short arms and medial lobe present. Aedeagus asymmetrical with basal part strongly sclerotized with short preatrium and large dorsal apodeme, the latter laterally compressed with distal anterior region membranous and distal posterior region strongly curved anteriorly; shaft elongate, with a single elongate apical process on one side; gonopore obscure.

Parazyginella resembles Zyginella Löw, but differs in its more greatly developed dorsal apodeme of the aedeagus and male pygofer with a dorsal finger-like process and without long macrosetae (Figs 16, 17).

Distribution. China (Guangxi, Yunnan).

\section{Key to species of Parazyginella}

$1 \quad$ Head without brown markings. Aedeagal shaft in lateral view similar in width throughout length, process moderately long and slim, lying parallel to shaft (Figs 10, 11) 
- $\quad$ Head with brown markings (Figs 2-4). Aedeagal shaft in lateral view slightly expanded from midlength to near apex, process long and stout, directed slightly towards basal apodeme (Figs 21, 22) P. tiani sp. n.

\section{Parazyginella lingtianensis Chou \& Zhang, 1985}

http://species-id.net/wiki/Parazyginella_lingtianensis

Figs 1, 5-12

Parazyginella lingtianensis Chou \& Zhang, 1985: 295; Zhang 1990: 174

Description. Head and pronotum whitish yellow. Scutellum and venter yellow; vertex and pronotum either side of midline marked with orange (Figs 1, 5); $3^{\text {rd }}$ apical cell of forewing with dark elliptical spot (Figs 1, 12).

Abdominal apodemes reaching middle part of 6th abdominal sternite (Fig. 6).

Subgenital plates with one macroseta near base, apex beak-like (Fig. 7). Paramere slightly expanded subapically (Fig. 8). Aedeagal shaft in lateral view similar in width throughout length, process moderately long and slim, lying parallel to shaft (Figs 10, 11).

Body length. Male $3.00 \mathrm{~mm}$ (including wing).

Material examined. Holotype, male, China: Guangxi Prov., Lingchuan, Lingtian, 5 June 1984, coll. Lu Xiaolin, lamp (NWAFU).

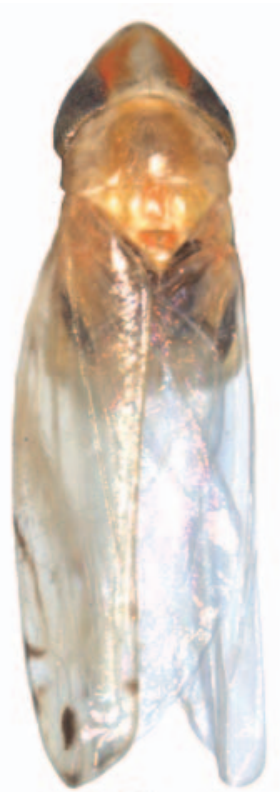

1

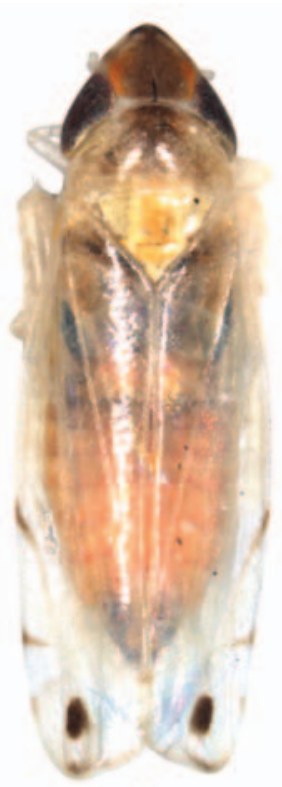

2

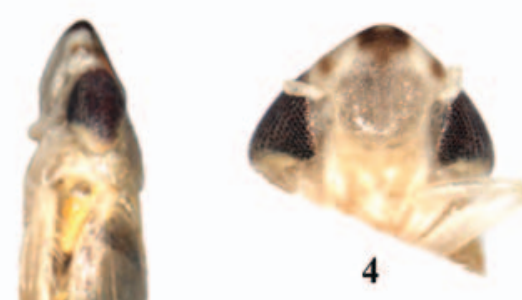

3

Figures I-4. I Parazyginella lingtianensis, dorsal habitus 2-4 Parazyginella tiani sp. n. 2 dorsal habitus 3 lateral habitus 4 face. 

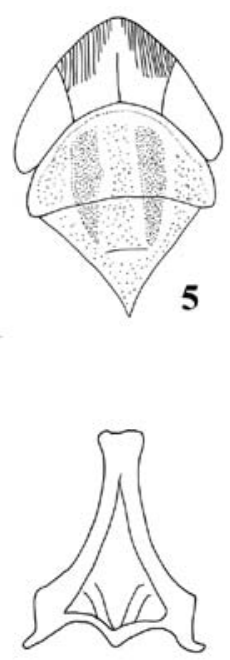

9
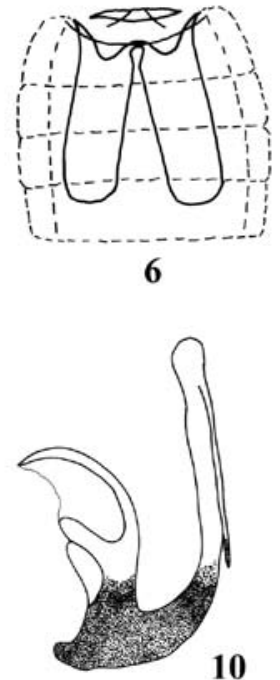
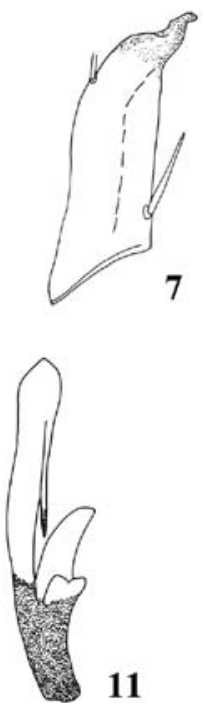
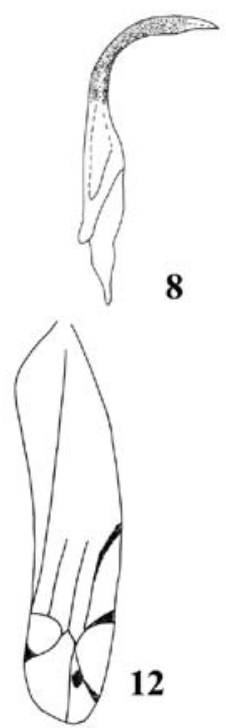

Figures 5-12. Parazyginella lingtianensis (after Chou and Zhang 1985) 5 Head, dorsal view 6 Abdominal apodeme 7 Subgenital plate 8 Paramere 9 Connective $\mathbf{0}$ Aedeagus, lateral view II Aedeagus, posterior view $\mathbf{I} \mathbf{2}$ Forewing.

Distribution. China (Guangxi).

Remarks. The male genitalia of the unique type could not be found. Therefore the pygofer (originally not figured) could not be examined and compared to the new species. Also, the original figure of the aedeagus (shown here, Fig. 10) did not show the membranous area of the basal apodeme shown in our new species. We conclude that this area was probably overlooked and add a line to the figure to show its approximate position.

\section{Parazyginella tiani sp. $\mathrm{n}$.}

urn:Isid:zoobank.org:act:A19A9268-1164-43C8-8B94-0C0CE69CBA7F http://species-id.net/wiki/Parazyginella_tiani

Figs 2-4, 13-22

Description. Head and pronotum whitish yellow; scutellum and venter yellow; vertex with disc and apex dark brown, orange laterally; face sordid white, with a dark brown patch above antennae; dorsum of abdomen stramineus. Forewing with dark spot in $3^{\text {rd }}$ apical cell (Figs 2, 3).

Abdominal apodemes nearly reaching end of 6th abdominal sternite (Fig. 14).

Male pygofer with short, sclerotized, sickle-like process caudo-dorsally (Figs 16, 17). Subgenital plates with two macrosetae near base, apex digitate with few microsetae (Figs 18,20). Paramere subapically with row of fine setae on outer margin and row of 


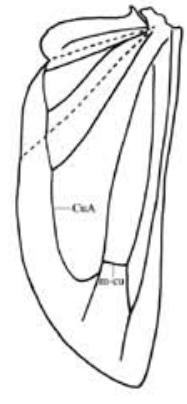

13

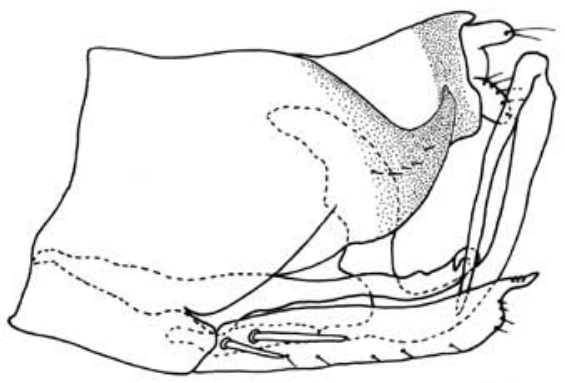

16

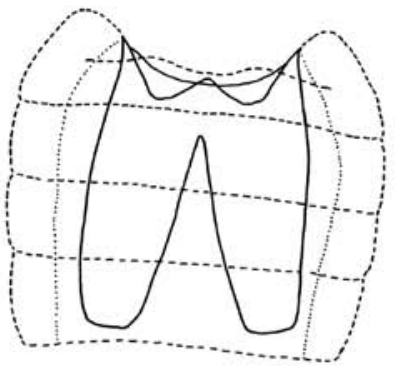

14

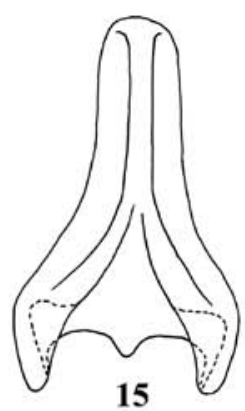

15

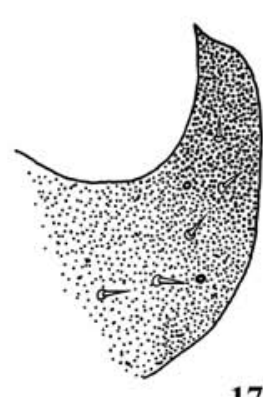

17
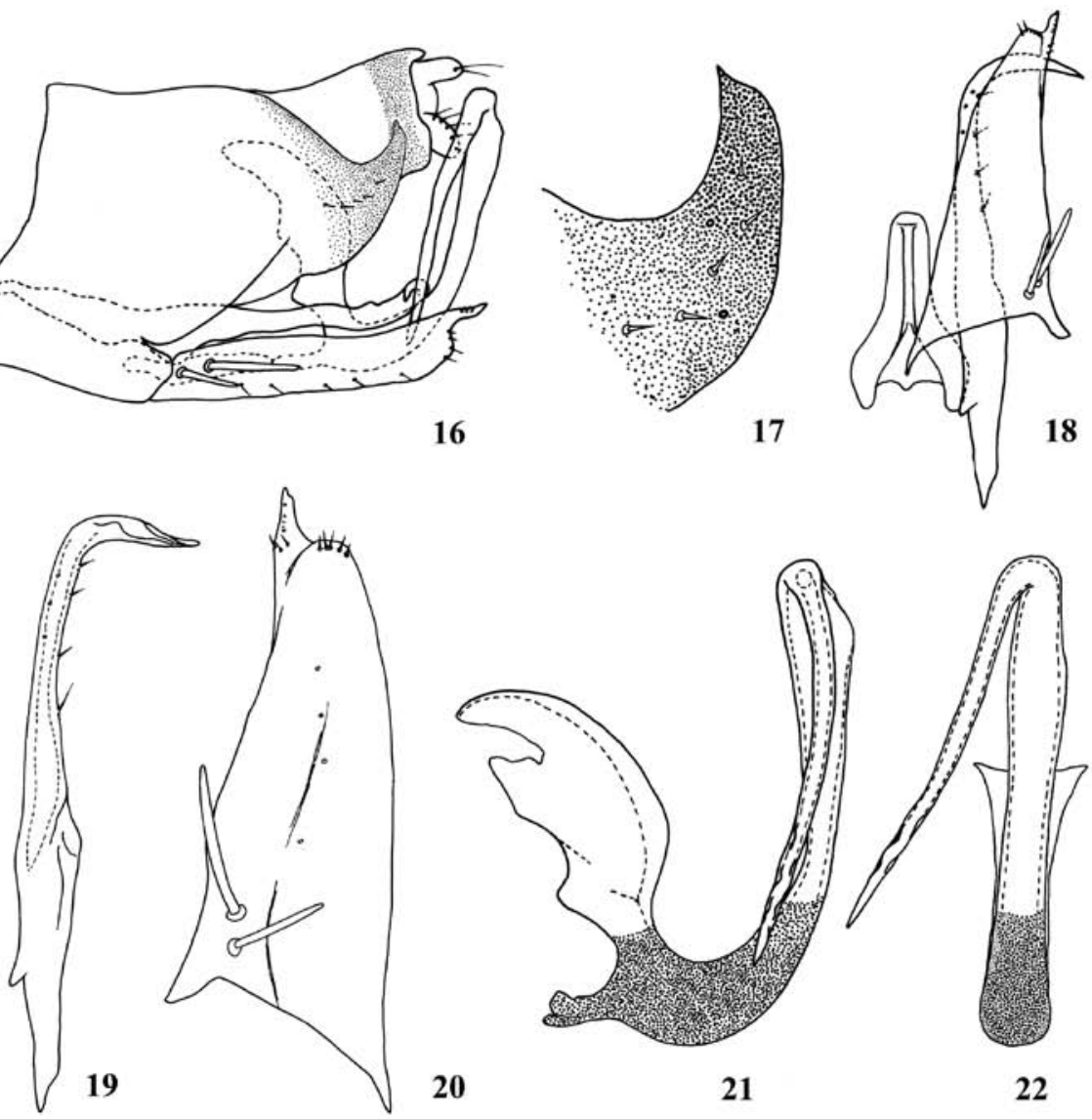

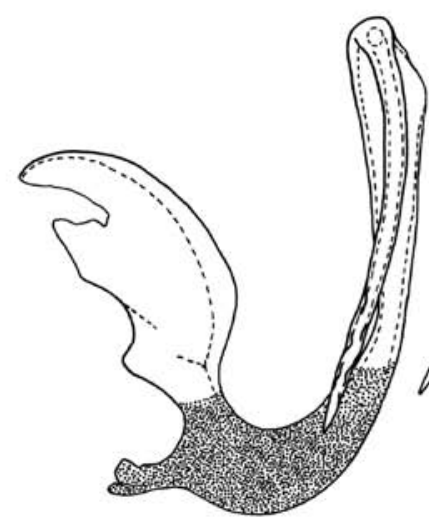

21

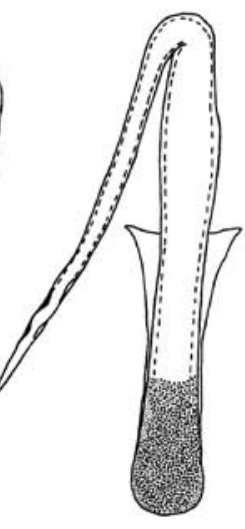

22

Figures 13-22. Parazyginella tiani sp. n. 13 Hindwing 14 Abdominal apodeme 15 Connective 16 Genital capsule, lateral view $\mathbf{1 7}$ Apical part of male pygofer 18 Paramere, connective, subgenital plate, ventral view 19 Paramere 20 Subgenital plate 21 Aedeagus, lateral view 22 Aedeagus, posterior view.

sensory pits on inner margin; with curved apical part with sinuate ridge (Figs 18, 19). Aedeagal shaft in lateral view slightly expanded from midlength to near apex, process long and stout, directed slightly towards basal apodeme, apex ornamented (Figs 21, 22). 
Body length. $2.95-2.98 \mathrm{~mm}$ (including wing).

Material examined. Holotype, male, China: Yunnan Province, Sanchahe, 7 June 1991, coll. Tian Rungang (NWAFU). Paratypes, two males, seven females, same data as holotype (NWAFU, BMNH).

Distribution. China (Yunnan).

Remarks. The new species resembles $P$. lingtianensis but differs in having brown markings on the vertex (compare Figs 1 and 2) and different shaped aedeagus as noted in the key.

Etymology. The new species is named after the collector's family name in gratitude.

\section{Acknowledgements}

We express our sincere thanks to Dr. I. Dworakowska (Canada) for her contribution to the knowledge of Chinese Typhlocybinae during her visit to the Entomological Museum, Northwest A \& F University, and revising the manuscript. We also appreciate Prof. Dr. J. R. Schrock (Emporia State University, USA), Mr M. Webb (The Natural History Museum, London, UK) for revising the manuscript. The research is supported by "The National Natural Science Foundation of China" (30770262), and "The Ministry of Education of China" (TS2011XBNL061), and also partly by "Basic Science and Technology Project of the Ministry of Science and Technology of China" (2006FY120100) and "Northwest A\&F University Grants for Outstanding Young Faculty Members” (Z111020905).

\section{Reference}

Chou I, Zhang YL (1985) On the tribe Zyginellini from China (Homoptera, Cicadellidae, Typhlocybinae ). Entomotaxonomia 7(4): 287-300.

Zhang YL (1990) A taxonomic study of Chinese Cicadellidae (Homoptera). Tianze Eldonejo, Yangling, Shaanxi, 174-176. 